\title{
The method of unitary clothing transformations in the theory of nucleon-nucleon scattering
}

\author{
I. Dubovyk ${ }^{1, \mathrm{a}}$ and A. Shebeko ${ }^{2, \mathrm{~b}}$ \\ 1 Institute of Electrophysics \& Radiation Technologies, \\ NAS of Ukraine, Kharkov, Ukraine \\ 2 NSC Kharkov Institute of Physics \& Technology \\ NAS of Ukraine, Kharkov, Ukraine
}

\begin{abstract}
The clothing procedure, put forward in quantum field theory (QFT) by Greenberg and Schweber, is applied for the description of nucleon-nucleon $(N-N)$ scattering. We consider pseudoscalar $(\pi$ and $\eta)$, vector $(\rho$ and $\omega)$ and scalar $(\delta$ and $\sigma$ ) meson fields interacting with $1 / 2 \operatorname{spin}(N$ and $\bar{N})$ fermion ones via the Yukawa-type couplings to introduce trial interactions between "bare" particles. The subsequent unitary clothing transformations (UCTs) are found to express the total Hamiltonian through new interaction operators that refer to particles with physical (observable) properties, the so-called clothed particles. In this work, we are focused upon the Hermitian and energy-independent operators for the clothed nucleons, being built up in the second order in the coupling constants. The corresponding analytic expressions in momentum space are compared with the separate meson contributions to the one-boson-exchange potentials in the meson theory of nuclear forces. In order to evaluate the $T$ matrix of the $N-N$ scattering we have used an equivalence theorem that enables us to operate in the clothed particle representation (CPR) instead of the bare particle representation (BPR) with its huge amount of virtual processes. We have derived the Lippmann-Schwinger(LS)-type equation for the CPR elements of the $T$-matrix for a given collision energy in the two-nucleon sector of the Hilbert space $\mathcal{H}$ of hadronic states and elaborated a code for its numerical solution in momentum space.
\end{abstract}

\section{Introductory remarks}

We know that there are a number of high precision, bosonexchange models of the two nucleon force $V_{N N}$, such as Paris [1], Bonn [2], Nijmegen [3], Argonne [4], CD Bonn [5] potentials and a fresh family of covariant oneboson-exchange (OBE) ones [6]. Note also successful treatments based on chiral effective field theory $[7,8]$, for a review see [9].

In this talk, we would like to draw attention to the first application of unitary clothing transformations $[10,11]$ in describing the nucleon-nucleon $(N-N)$ scattering. Recall that such transformations $W$, being aimed at the inclusion of the so-called cloud or persistent effects, make it possible the transition from the bare-particle representation (BPR) to the clothed-particle representation (CPR) in the Hilbert space $\mathcal{H}$ of meson-nucleon states. In this way, a large amount of virtual processes induced with the meson absorption/emission, the $N \bar{N}$-pair annihilation/production and other cloud effects can be accumulated in the creation (destruction) operators $\alpha_{c}$ for the "clothed" (physical) mesons and nucleons. Such a bootstrap reflects the most significant distinction between the concepts of clothed and bare particles.
In the course of the clothing procedure all the generators of the Poincare group get one and the same sparse structure on $\mathcal{H}[10]$. Here we will focus upon one of them, viz., the total Hamiltonian

$$
H=H_{F}(\alpha)+H_{I}(\alpha) \equiv H(\alpha)
$$

with

$$
H_{I}(\alpha)=V(\alpha)+\text { mass and vertex counterterms, }
$$

where free part $H_{F}(\alpha)$ and interaction $V(\alpha)$ depend on creation (destruction) operators $\alpha^{\dagger}(\alpha)$ in the BPR , i.e., referred to bare particles with physical masses [11], where they have been introduced via the mass-changing Bogoliubov-type UTs. To be more definite, let us consider fermions (nucleons and antinucleons) and bosons $\left(\pi-, \eta_{-}, \rho-, \omega-\right.$ mesons, etc.) interacting via the Yukawa-type couplings for scalar (s), pseudoscalar (ps) and vector (v) mesons (see, e.g., [2]). Then, using a trick prompted by the derivation of Eq. (7.5.22) in [12] to eliminate in a proper way the vectorfield component $\varphi_{\mathrm{v}}^{0}$, we have $V(\alpha)=V_{s}+V_{p s}+V_{\mathrm{v}}$ with

$$
\begin{gathered}
V_{s}=g_{s} \int d \mathbf{x} \bar{\psi}(\mathbf{x}) \psi(\mathbf{x}) \varphi_{s}(\mathbf{x}) \\
V_{p s}=i g_{p s} \int d \mathbf{x} \bar{\psi}(\mathbf{x}) \gamma_{5} \psi(\mathbf{x}) \varphi_{p s}(\mathbf{x})
\end{gathered}
$$

\footnotetext{
a e-mail: e.a.dubovyk@mail.ru

b e-mail: shebeko@kipt.kharkov.ua
} 


$$
\begin{array}{r}
V_{\mathrm{v}}=\int d \mathbf{x}\left\{g_{\mathrm{v}} \bar{\psi}(\mathbf{x}) \gamma_{\mu} \psi(\mathbf{x}) \varphi_{\mathrm{v}}^{\mu}(\mathbf{x})\right. \\
\left.+\frac{f_{\mathrm{v}}}{4 m} \bar{\psi}(\mathbf{x}) \sigma_{\mu \nu} \psi(\mathbf{x}) \varphi_{\mathrm{v}}^{\mu v}(\mathbf{x})\right\} \\
+\int d \mathbf{x}\left\{\frac{g_{\mathrm{v}}^{2}}{2 m_{\mathrm{v}}^{2}} \bar{\psi}(\mathbf{x}) \gamma_{0} \psi(\mathbf{x}) \bar{\psi}(\mathbf{x}) \gamma_{0} \psi(\mathbf{x})\right. \\
\left.+\frac{f_{\mathrm{v}}^{2}}{4 m^{2}} \bar{\psi}(\mathbf{x}) \sigma_{0 i} \psi(\mathbf{x}) \bar{\psi}(\mathbf{x}) \sigma_{0 i} \psi(\mathbf{x})\right\},
\end{array}
$$

with the boson fields $\varphi_{b}$ and the fermion field $\psi$, where $\varphi_{\mathrm{v}}^{\mu \nu}(\mathbf{x})=\partial^{\mu} \varphi_{\mathrm{v}}^{v}(\mathbf{x})-\partial^{v} \varphi_{\mathrm{v}}^{\mu}(\mathbf{x})$ the tensor of the vector field included. The mass (vertex) counterterms are given by Eqs. (32)-(33) of Ref. [11] (the difference $V_{0}(\alpha)-V(\alpha)$ where a primary interaction $V_{0}(\alpha)$ is derived from $V(\alpha)$ replacing the "physical" coupling constants by "bare" ones).

The corresponding set $\alpha$ involves operators $a^{\dagger}(a)$ for the bosons, $b^{\dagger}(b)$ for the nucleons and $d^{\dagger}(d)$ for the antinucleons. In their terms, e.g., we have the free pion and fermion parts

$$
\begin{aligned}
& H_{F}(\alpha)=\int d \mathbf{k} \omega_{\mathbf{k}} a^{\dagger}(\mathbf{k}) a(\mathbf{k}) \\
& +\int d \mathbf{p} E_{\mathbf{p}} \sum_{\mu}\left[b^{\dagger}(\mathbf{p}, \mu) b(\mathbf{p}, \mu)+d^{\dagger}(\mathbf{p}, \mu) d(\mathbf{p}, \mu)\right]
\end{aligned}
$$

and the primary trilinear interaction

$$
V(\alpha) \sim a b^{\dagger} b+a b^{\dagger} d^{\dagger}+a d b+a d d^{\dagger}+\text { H.c. }
$$

with the three-legs vertices. Here $\omega_{\mathbf{k}}=\sqrt{m_{b}^{2}+\mathbf{k}^{2}}\left(E_{\mathbf{p}}=\right.$ $\sqrt{m^{2}+\mathbf{p}^{2}}$ ) the pion (nucleon) energy with physical mass $m_{b}(m), \mu$ the fermion polarization index.

We have tried to draw parallels with that field-theoretic background which has been employed in boson-exchange models. First of all, we imply the approach by the Bonn group [2,5], where, following the idea by Schütte [13], the authors started from the total Hamiltonian (in our notations),

$$
H=H_{F}(\alpha)+V(\alpha)
$$

with the boson-nucleon interaction

$$
V(\alpha) \sim a b^{\dagger} b+H . c .
$$

\section{Analytic expressions for the quasipotentials in momentum space}

As shown in [10], after eliminating the so-called bad terms ${ }^{1}$ from $V(\alpha)$ the primary Hamiltonian $H(\alpha)$ can be represented in the form,

$$
H(\alpha)=K_{F}\left(\alpha_{c}\right)+K_{I}\left(\alpha_{c}\right) \equiv K\left(\alpha_{c}\right)
$$

\footnotetext{
${ }^{1}$ By definition, they prevent the bare vacuum $\Omega_{0}\left(a\left|\Omega_{0}\right\rangle=\right.$ $\left.b\left|\Omega_{0}\right\rangle=\ldots=0\right)$ and the bare one-particle states $\mid$ 1bare $\rangle \equiv a^{\dagger}\left|\Omega_{0}\right\rangle$ $\left(b^{\dagger}\left|\Omega_{0}\right\rangle, \ldots\right)$ to be $H$ eigenstates.
}

The free part of the new decomposition is determined by

$$
\begin{aligned}
& K_{F}\left(\alpha_{c}\right)=\int d \mathbf{k} \omega_{\mathbf{k}} a_{c}^{\dagger}(\mathbf{k}) a_{c}(\mathbf{k}) \\
& +\int d \mathbf{p} E_{\mathbf{p}} \sum_{\mu}\left[b_{c}^{\dagger}(\mathbf{p}, \mu) b_{c}(\mathbf{p}, \mu)+d_{c}^{\dagger}(\mathbf{p}, \mu) d_{c}(\mathbf{p}, \mu)\right]
\end{aligned}
$$

while $K_{I}$ contains only interactions responsible for physical processes, these quasipotentials between the clothed particles, e.g.,

$$
\begin{array}{r}
K_{I}^{(2)}\left(\alpha_{c}\right)=K(N N \rightarrow N N)+K(\bar{N} \bar{N} \rightarrow \bar{N} \bar{N}) \\
+K(N \bar{N} \rightarrow N \bar{N})+K(b N \rightarrow b N)+K(b \bar{N} \rightarrow b \bar{N}) \\
+K(b b \rightarrow N \bar{N})+K(N \bar{N} \rightarrow b b)
\end{array}
$$

In accordance with the clothing procedure developed in [10] they obey the following requirements:

i) The physical vacuum (the $H$ lowest eigenstate) must coincide with a new no-particle state $\Omega$, i.e., the state that obeys the equations

$$
\begin{gathered}
a_{c}(\mathbf{k})|\Omega\rangle=b_{c}(\mathbf{p}, \mu)|\Omega\rangle=d_{c}(\mathbf{p}, \mu)|\Omega\rangle=0, \\
\forall \mathbf{k}, \mathbf{p}, \mu \quad(\langle\Omega \mid \Omega\rangle=1) .
\end{gathered}
$$

ii) New one-clothed-particle states $|\mathbf{k}\rangle_{c} \equiv a_{c}^{\dagger}(\mathbf{k}) \Omega$ etc. are the eigenvectors both of $K_{F}$ and $K$,

$$
\begin{gathered}
K\left(\alpha_{c}\right)|\mathbf{k}\rangle_{c}=K_{F}\left(\alpha_{c}\right)|\mathbf{k}\rangle_{c}=\omega_{k}|\mathbf{k}\rangle_{c} \\
K_{I}\left(\alpha_{c}\right)|\mathbf{k}\rangle_{c}=0
\end{gathered}
$$

iii) The spectrum of indices that enumerate the new operators must be the same as that for the bare ones .

iv) The new operators $\alpha_{c}$ satisfy the same commutation rules as do their bare counterparts $\alpha$, since the both sets are connected to each other via the similarity transformation

$$
\alpha_{c}=W^{\dagger} \alpha W,
$$

with a unitary operator $W$ to be obtained as in [10].

It is important to realize that operator $K\left(\alpha_{c}\right)$ is the same Hamiltonian $H(\alpha)$. Accordingly $[10,11]$ the $N-N$ interaction operator in the CPR has the following structure:

$$
K(N N \rightarrow N N)=\sum_{b} K_{b}(N N \rightarrow N N),
$$

$$
\begin{aligned}
K_{b}(N N \rightarrow & N N)=\int \sum_{\mu} d \mathbf{p}_{1}^{\prime} d \mathbf{p}_{2}^{\prime} d \mathbf{p}_{1} d \mathbf{p}_{2} \\
& \times V_{b}\left(1^{\prime}, 2^{\prime} ; 1,2\right) b_{c}^{\dagger}\left(1^{\prime}\right) b_{c}^{\dagger}\left(2^{\prime}\right) b_{c}(1) b_{c}(2),
\end{aligned}
$$

where the symbol $\sum_{\mu}$ denotes the summation over nucleon spin projections, $1=\left\{\mathbf{p}_{1}, \mu_{1}\right\}$, etc.

For our evaluations of the c-number matrices $V_{b}$ we have employed some experience from Refs. [10,11] to get in the second order in the coupling constants

$$
\begin{aligned}
V_{b}\left(1^{\prime}, 2^{\prime} ; 1,2\right) & =\frac{1}{(2 \pi)^{3}} \frac{m^{2}}{\sqrt{E_{\mathbf{p}_{1}^{\prime}} E_{\mathbf{p}_{2}^{\prime}} E_{\mathbf{p}_{1}} E_{\mathbf{p}_{2}}}} \\
\times & \delta\left(\mathbf{p}_{1}^{\prime}+\mathbf{p}_{2}^{\prime}-\mathbf{p}_{1}-\mathbf{p}_{2}\right) v_{b}\left(1^{\prime}, 2^{\prime} ; 1,2\right),
\end{aligned}
$$


$19^{\text {th }}$ International IUPAP Conference on Few-Body Problems in Physics

$$
\begin{aligned}
& v_{s}\left(1^{\prime}, 2^{\prime} ; 1,2\right) \\
& =-\frac{g_{s}^{2}}{2} \bar{u}\left(\mathbf{p}_{1}^{\prime}\right) u\left(\mathbf{p}_{1}\right) \frac{1}{\left(p_{1}-p_{1}^{\prime}\right)^{2}-m_{s}^{2}} \bar{u}\left(\mathbf{p}_{2}^{\prime}\right) u\left(\mathbf{p}_{2}\right), \\
& v_{p s}\left(1^{\prime}, 2^{\prime} ; 1,2\right) \\
& =\frac{g_{p s}^{2}}{2} \bar{u}\left(\mathbf{p}_{1}^{\prime}\right) \gamma_{5} u\left(\mathbf{p}_{1}\right) \frac{1}{\left(p_{1}-p_{1}^{\prime}\right)^{2}-m_{p s}^{2}} \bar{u}\left(\mathbf{p}_{2}^{\prime}\right) \gamma_{5} u\left(\mathbf{p}_{2}\right), \\
& v_{\mathrm{v}}\left(1^{\prime}, 2^{\prime} ; 1,2\right)=\frac{1}{2} \frac{1}{\left(p_{1}^{\prime}-p_{1}\right)^{2}-m_{\mathrm{v}}^{2}} \\
& \times\left[\bar{u}\left(\mathbf{p}_{1}^{\prime}\right)\left\{\left(g_{\mathrm{v}}+f_{\mathrm{v}}\right) \gamma_{v}-\frac{f_{\mathrm{v}}}{2 m}\left(p_{1}^{\prime}+p_{1}\right)_{v}\right\} u\left(\mathbf{p}_{1}\right)\right. \\
& \quad \times \bar{u}\left(\mathbf{p}_{2}^{\prime}\right)\left\{\left(g_{\mathrm{v}}+f_{\mathrm{v}}\right) \gamma^{v}-\frac{f_{\mathrm{v}}}{2 m}\left(p_{2}^{\prime}+p_{2}\right)^{v}\right\} u\left(\mathbf{p}_{2}\right) \\
& -\bar{u}\left(\mathbf{p}_{1}^{\prime}\right)\left\{\left(g_{\mathrm{v}}+f_{\mathrm{v}}\right) \gamma_{v}-\frac{f_{\mathrm{v}}}{2 m}\left(p_{1}^{\prime}+p_{1}\right)_{v}\right\} u\left(\mathbf{p}_{1}\right) \\
& \quad \times \bar{u}\left(\mathbf{p}_{2}^{\prime}\right) \frac{f_{\mathrm{v}}}{2 m}\left\{\left(\hat{p}_{1}^{\prime}+\hat{p}_{2}^{\prime}-\hat{p}_{1}-\hat{p}_{2}\right) \gamma^{v}\right. \\
& \left.\left.\quad-\left(p_{1}^{\prime}+p_{2}^{\prime}-p_{1}-p_{2}\right)^{v}\right\} u\left(\mathbf{p}_{2}\right)\right],
\end{aligned}
$$

where $m_{b}$ the mass of the clothed boson (its physical value) and $\hat{q}=q_{\mu} \gamma^{\mu}$. In the framework of the isospin formalism one needs to add the factor $\tau(1) \tau(2)$ in the corresponding expressions.

At this point, our derivation of the vector-boson contribution (21) is to be specifically commented. Actually, it is the case, where for a Lorentz-invariant Lagrangian it is not necessarily to have "... the interaction Hamiltonian as the integral over space of a scalar interaction density; we also need to add non-scalar terms to the interaction density ..." (quoted from p.292 of Ref. [12]). Let us recall that the density in question has the property,

$$
U_{F}(\Lambda, a) \mathcal{H}(x) U_{F}^{-1}(\Lambda, a)=\mathcal{H}(\Lambda x+a),
$$

where the operators $U_{F}(\Lambda, a)$ realize a unitary irreducible representation of the Poincare group in the Hilbert space of states for free (non-interacting) fields.

By definition, the first clothing transformation $W^{(1)}=$ $\exp \left[R^{(1)}\right]\left(R^{(1)^{\dagger}}=-R^{(1)}\right)$ eliminates all interactions linear in the coupling constants, viz.,

$$
V^{(1)}=V_{s}+V_{p s}+V_{\mathrm{v}}^{(1)}
$$

with

$$
\begin{aligned}
V_{\mathrm{v}}^{(1)}=\int d \mathbf{x}\left\{g_{\mathrm{v}} \bar{\psi}(\mathbf{x}) \gamma_{\mu} \psi(\mathbf{x}) \varphi_{\mathrm{v}}^{\mu}(\mathbf{x})\right. & \\
& \left.+\frac{f_{\mathrm{v}}}{4 m} \bar{\psi}(\mathbf{x}) \sigma_{\mu \nu} \psi(\mathbf{x}) \varphi_{\mathrm{v}}^{\mu \nu}(\mathbf{x})\right\} .
\end{aligned}
$$

Following Ref.[10] we have

$$
R^{(1)}=-i \lim _{\varepsilon \rightarrow 0+} \int_{0}^{\infty} V_{D}^{(1)}(t) e^{-\varepsilon t} d t
$$

if $m_{b}<2 m$. Here

$$
V_{D}^{(1)} \equiv \exp \left[i H_{F} t\right] V^{(1)} \exp \left[-i H_{F} t\right]=\int \mathcal{H}^{(1)}(x) d \mathbf{x},
$$

where $\mathcal{H}^{(1)}(x)$ is the Lorentz scalar.

The corresponding interaction operator in the CPR (12) can be written as

$$
K_{I}^{(2)}\left(\alpha_{c}\right)=\frac{1}{2}\left[R^{(1)}\left(\alpha_{c}\right), V^{(1)}\left(\alpha_{c}\right)\right]+V^{(2)}\left(\alpha_{c}\right),
$$

where we have kept only the contributions of the second order in the coupling constants, so

$$
\begin{aligned}
V^{(2)}=\int d \mathbf{x}\left\{\frac{g_{\mathrm{v}}^{2}}{2 m_{\mathrm{v}}^{2}} \bar{\psi}(x) \gamma_{0} \psi(\mathbf{x}) \bar{\psi}(\mathbf{x}) \gamma_{0} \psi(\mathbf{x})\right. \\
\left.+\frac{f_{\mathrm{v}}^{2}}{4 m^{2}} \bar{\psi}(\mathbf{x}) \sigma_{0 i} \psi(\mathbf{x}) \bar{\psi}(\mathbf{x}) \sigma_{0 i} \psi(\mathbf{x})\right\} .
\end{aligned}
$$

We point out that all quantities in the r.h.s. of Eq.(25) depend on the new creation(destruction) operators $\alpha_{c}$. In particular, it means that in the standard Fourier expansions of the fields involved in the definitions of $V^{(1)}$ and $V^{(2)}$ one should replace the set $\{\alpha\}$ by the set $\left\{\alpha_{c}\right\}$. Thus, there is an essential distinction between $V^{(1)}\left(V^{(2)}\right)$, on the one hand, and the first(second) integral in the r.h.s. of Eq.(5), on the other hand.

For this exposition we do not intend to derive all interactions between the clothed mesons and nucleons, allowed by formula (25). Our aim is more humble, viz., to find in the r.h.s. of Eq.(25) terms of the type (17), responsible for the $N-N$ interaction. Meanwhile, in case of the vector mesons we encounter an interplay between the commutator $\left[R_{\mathrm{v}}^{(1)}, V_{\mathrm{v}}^{(1)}\right] / 2$ and the integral (26). Indeed, after a simple algebra we find

$$
\begin{aligned}
\frac{1}{2}\left[R^{(1)}, V^{(1)}\right]_{\mathrm{v}} & (N N \rightarrow N N) \\
= & K_{\mathrm{v}}(N N \rightarrow N N)+K_{\text {cont }}(N N \rightarrow N N),
\end{aligned}
$$

where the first term has the structure of Eq.(17) with the coefficients by (21). At the same time the second term $K_{\text {cont }}$ completely cancels the non-scalar operator $V^{(2)}$. The latter may be associated with a contact interaction since it does not contain any propagators (cf. the approach by the Osaka group [14]), being expressed through the $b_{c}^{\dagger}\left(b_{c}\right)$. In other words, the first UCT enables us to remove the noninvariant terms directly in the Hamiltonian. In our opinion, such a cancellation, first discussed here, is a pleasant feature of the CPR.

Moreover, as it was shown in Ref.[10], for each boson included the corresponding relativistic and properly symmetrized $N-N$ interaction, the kernel of integral equations for the $N-N$ bound and scattering states, is determined by

$$
\begin{array}{r}
\left\langle b_{c}^{\dagger}\left(\mathbf{p}_{1}^{\prime}\right) b_{c}^{\dagger}\left(\mathbf{p}_{2}^{\prime}\right) \Omega\left|K_{b}(N N \rightarrow N N)\right| b_{c}^{\dagger}\left(\mathbf{p}_{1}\right) b_{c}^{\dagger}\left(\mathbf{p}_{2}\right) \Omega\right\rangle \\
=V_{b}^{\text {dir }}\left(1^{\prime}, 2^{\prime} ; 1,2\right)-V_{b}^{\text {exc }}\left(1^{\prime}, 2^{\prime} ; 1,2\right),
\end{array}
$$


where we have separated the so-called direct

$$
V_{b}^{d i r}\left(1^{\prime}, 2^{\prime} ; 1,2\right)=-V_{b}\left(1^{\prime}, 2^{\prime} ; 1,2\right)-V_{b}\left(2^{\prime}, 1^{\prime} ; 2,1\right)
$$

and exchange

$$
V_{b}^{e x c}\left(1^{\prime}, 2^{\prime} ; 1,2\right)=V_{b}^{d i r}\left(2^{\prime}, 1^{\prime} ; 1,2\right)
$$

terms. For example, the one-pion-exchange contribution can be divided into the two parts:

$$
\begin{aligned}
V_{\pi}^{d i r}\left(1^{\prime}, 2^{\prime} ; 1,2\right)=-\frac{g_{\pi}^{2}}{(2 \pi)^{3}} \frac{m^{2}}{\sqrt{E_{\mathbf{p}_{1}^{\prime}} E_{\mathbf{p}_{2}^{\prime}} E_{\mathbf{p}_{1}} E_{\mathbf{p}_{2}}}} \\
\times \delta\left(\mathbf{p}_{1}^{\prime}+\mathbf{p}_{2}^{\prime}-\mathbf{p}_{1}-\mathbf{p}_{2}\right) \bar{u}\left(\mathbf{p}_{1}^{\prime}\right) \gamma_{5} u\left(\mathbf{p}_{1}\right) \bar{u}\left(\mathbf{p}_{2}^{\prime}\right) \gamma_{5} u\left(\mathbf{p}_{2}\right) \\
\quad \times \frac{1}{2}\left\{\frac{1}{\left(p_{1}-p_{1}^{\prime}\right)^{2}-m_{\pi}^{2}}+\frac{1}{\left(p_{2}-p_{2}^{\prime}\right)^{2}-m_{\pi}^{2}}\right\}
\end{aligned}
$$

and

$$
\begin{gathered}
V_{\pi}^{\operatorname{exc}}\left(1^{\prime}, 2^{\prime} ; 1,2\right)=-\frac{g_{\pi}^{2}}{(2 \pi)^{3}} \frac{m^{2}}{\sqrt{E_{\mathbf{p}_{1}^{\prime}} E_{\mathbf{p}_{2}^{\prime}} E_{\mathbf{p}_{1}} E_{\mathbf{p}_{2}}}} \\
\times \delta\left(\mathbf{p}_{1}^{\prime}+\mathbf{p}_{2}^{\prime}-\mathbf{p}_{1}-\mathbf{p}_{2}\right) \bar{u}\left(\mathbf{p}_{1}^{\prime}\right) \gamma_{5} u\left(\mathbf{p}_{2}\right) \bar{u}\left(\mathbf{p}_{2}^{\prime}\right) \gamma_{5} u\left(\mathbf{p}_{1}\right) \\
\quad \times \frac{1}{2}\left\{\frac{1}{\left(p_{2}-p_{1}^{\prime}\right)^{2}-m_{\pi}^{2}}+\frac{1}{\left(p_{1}-p_{2}^{\prime}\right)^{2}-m_{\pi}^{2}}\right\}
\end{gathered}
$$

to be depicted in Fig.1, where the dashed lines correspond to the following Feynman-like "propagators":

$$
\frac{1}{2}\left\{\frac{1}{\left(p_{1}-p_{1}^{\prime}\right)^{2}-m_{\pi}^{2}}+\frac{1}{\left(p_{2}-p_{2}^{\prime}\right)^{2}-m_{\pi}^{2}}\right\}
$$

on the left panel and

$$
\frac{1}{2}\left\{\frac{1}{\left(p_{2}-p_{1}^{\prime}\right)^{2}-m_{\pi}^{2}}+\frac{1}{\left(p_{1}-p_{2}^{\prime}\right)^{2}-m_{\pi}^{2}}\right\}
$$

on the right panel. Other distinctive features of the result (27) have been discussed in $[10,11]$.
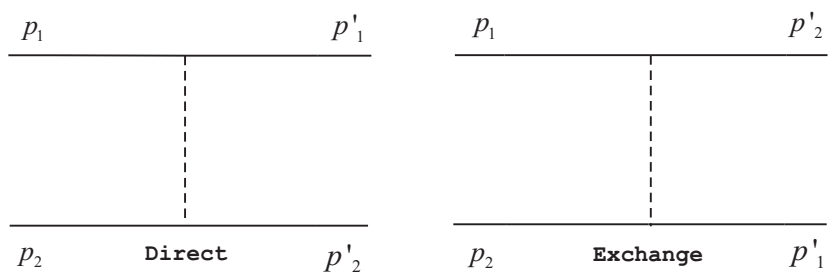

Fig. 1. The Feynman-like diagrams for the direct and exchange contributions in the r.h.s. of Eq.(27).

\section{The field-theoretic description of the elastic $\mathbf{N}-\mathbf{N}$ scattering}

\subsection{The $T$-matrix in the CPR}

In order to evaluate the $N-N$ scattering amplitude for the collision energy $E$ we will regard a field operator $T$ that meets the equation

$$
T(E+i 0)=H_{I}+H_{I}\left(E+i 0-H_{F}\right)^{-1} T(E+i 0)
$$

and whose matrix elements $\langle N N|T(E+i 0)| N N\rangle$ on the energy shell $E=E_{1}+E_{2}=E_{1}^{\prime}+E_{2}^{\prime}$ can be expressed through the phase shifts and mixing parameters.

Unlike nonrelativistic quantum mechanics (NQM) in relativistic QFT the interaction $H_{I}$ does not conserve the particle number, being the spring of particle creation and destruction. The feature makes the problem of finding the $N-N$ scattering matrix much more complicated than in the framework of nonrelativistic approach.

Such a general field-theoretic consideration can be simplified with the help of an equivalence theorem [15] according to which the $S$ matrix elements in the Dirac (D) picture, viz.,

$$
S_{f i} \equiv\left\langle\alpha^{\dagger} \ldots \Omega_{0}|S(\alpha)| \alpha^{\dagger} \ldots \Omega_{0}\right\rangle
$$

are equal to the corresponding elements

$$
S_{f i}^{c} \equiv\left\langle\alpha_{c}^{\dagger} \ldots \Omega\left|S\left(\alpha_{c}\right)\right| \alpha_{c}^{\dagger} \ldots \Omega\right\rangle
$$

of the $S$ matrix in the CPR once the UCTs

$$
W_{D}(t)=\exp \left(i K_{F} t\right) W \exp \left(-i K_{F} t\right)
$$

obey the condition

$$
W_{D}( \pm \infty)=1
$$

The $T$ operator in the CPR satisfies the equation

$$
\begin{aligned}
T_{\text {cloth }}(E+i 0)= & K_{I} \\
& +K_{I}\left(E+i 0-K_{F}\right)^{-1} T_{\text {cloth }}(E+i 0)
\end{aligned}
$$

and the matrix

$$
\begin{aligned}
T_{f i} \equiv\langle f ; b|T(E+i 0)| i ; b\rangle \\
\quad=\left\langle f ; c\left|T_{\text {cloth }}(E+i 0)\right| i ; c\right\rangle \equiv T_{f i}^{c},
\end{aligned}
$$

where $|; b\rangle(|; c\rangle)$ are the $H_{F}\left(K_{F}\right)$ eigenvectors, may be evaluated relying upon properties of the new interaction $K_{I}\left(\alpha_{c}\right)$.

If in Eq.(36) we approximate $K_{I}$ by $K_{I}^{(2)}$, then initial task of evaluating the CPR matrix elements can be reduced to solving the equation

$$
\begin{aligned}
& \left\langle 1^{\prime}, 2^{\prime}\left|T_{N N}(E)\right| 1,2\right\rangle=\left\langle 1^{\prime}, 2^{\prime}\left|K_{N N}\right| 1,2\right\rangle \\
& +\left\langle 1^{\prime}, 2^{\prime}\left|K_{N N}\left(E+i 0-K_{F}\right)^{-1} T_{N N}(E)\right| 1,2\right\rangle .
\end{aligned}
$$

\subsection{The $R$-matrix equation and its angular-momentum decomposition}

For practical applications one prefers to work with the corresponding $R$-matrix that meets the set of equations

$$
\begin{aligned}
\left\langle 1^{\prime} 2^{\prime}|\bar{R}(E)| 12\right\rangle & =\left\langle 1^{\prime} 2^{\prime}\left|\bar{K}_{N N}\right| 12\right\rangle \\
+ & \int_{34}\left\langle 1^{\prime} 2^{\prime}\left|\bar{K}_{N N}\right| 34\right\rangle \frac{\langle 34|\bar{R}(E)| 12\rangle}{E-E_{3}-E_{4}}
\end{aligned}
$$


$19^{\text {th }}$ International IUPAP Conference on Few-Body Problems in Physics

with $\bar{R}(E)=R(E) / 2$ and $\bar{K}_{N N}=K_{N N} / 2$, where the oper-

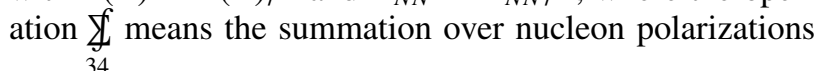
and the p.v. integration over nucleon momenta. The kernel of Eq.(39) is

$$
\begin{aligned}
\left\langle 1^{\prime} 2^{\prime}\left|\bar{K}_{N N}\right| 12\right\rangle & =\delta\left(\mathbf{p}_{1}^{\prime}+\mathbf{p}_{2}^{\prime}-\mathbf{p}_{1}-\mathbf{p}_{2}\right)\left\langle 1^{\prime} 2^{\prime}|\bar{V}| 12\right\rangle \\
\equiv \delta\left(\mathbf{p}_{1}^{\prime}+\mathbf{p}_{2}^{\prime}-\right. & \left.\mathbf{p}_{1}-\mathbf{p}_{2}\right) \\
& \times\left\langle\mathbf{p}_{1}^{\prime} \mu_{1}^{\prime} \tau_{1}^{\prime}, \mathbf{p}_{2}^{\prime} \mu_{2}^{\prime} \tau_{2}^{\prime}|\bar{V}| \mathbf{p}_{1} \mu_{1} \tau_{1}, \mathbf{p}_{2} \mu_{2} \tau_{2}\right\rangle
\end{aligned}
$$

The subsequent calculations are essentially simplified in the center-of-mass system (c.m.s) in which

$$
\begin{array}{r}
\left\langle\mathbf{p}^{\prime} \mu_{1}^{\prime} \mu_{2}^{\prime}, \tau_{1}^{\prime} \tau_{2}^{\prime}|\bar{R}(E)| \mathbf{p} \mu_{1} \mu_{2}, \tau_{1} \tau_{2}\right\rangle \\
=\left\langle\mathbf{p}^{\prime} \mu_{1}^{\prime} \mu_{2}^{\prime}, \tau_{1}^{\prime} \tau_{2}^{\prime}|\bar{V}| \mathbf{p} \mu_{1} \mu_{2}, \tau_{1} \tau_{2}\right\rangle \\
+\sum p . v . \int d \mathbf{q}\left\langle\mathbf{p}^{\prime} \mu_{1}^{\prime} \mu_{2}^{\prime}, \tau_{1}^{\prime} \tau_{2}^{\prime}|\bar{V}| \mathbf{q} \mu_{3} \mu_{4}, \tau_{3} \tau_{4}\right\rangle \\
\times \frac{\left\langle\mathbf{q} \mu_{3} \mu_{4}, \tau_{3} \tau_{4}|\bar{R}(E)| \mathbf{p} \mu_{1} \mu_{2}, \tau_{1} \tau_{2}\right\rangle}{E-2 E_{\mathbf{q}}}
\end{array}
$$

Here the quantum numbers $\mu(\tau)$ are the individual spin (isospin) projections.

Accordingly Eq. (27)

$$
\begin{aligned}
&\left\langle 1^{\prime} 2^{\prime}|\bar{V}| 12\right\rangle=\frac{1}{2(2 \pi)^{3}} \frac{m^{2}}{E_{\mathbf{p}^{\prime}} E_{\mathbf{p}}} \\
& \times \sum_{b}\left[v_{b}^{d i r}\left(1^{\prime}, 2^{\prime} ; 1,2\right)-v_{b}^{e x c}\left(1^{\prime}, 2^{\prime} ; 2,1\right)\right]
\end{aligned}
$$

with

$$
v_{b}^{d i r}\left(1^{\prime}, 2^{\prime} ; 1,2\right)=-v_{b}\left(1^{\prime}, 2^{\prime} ; 1,2\right)-v_{b}\left(2^{\prime}, 1^{\prime} ; 2,1\right)
$$

and

$$
v_{b}^{e x c}\left(1^{\prime}, 2^{\prime} ; 2,1\right)=v_{b}^{d i r}\left(2^{\prime}, 1^{\prime} ; 1,2\right),
$$

where the separate boson contributions are determined by Eqs. (19)-(21) with $\mathbf{p}_{1}=\mathbf{p}=-\mathbf{p}_{2}$ and $\mathbf{p}_{1}^{\prime}=\mathbf{p}^{\prime}=-\mathbf{p}_{2}^{\prime}$.

Following a common practice we are interested in the angular-momentum decomposition of Eq.(40) assuming a nonrelativistic analog of relativistic partial wave expansions (see [16] and refs. therein) for two-particle states. For example, the clothed two-nucleon state (the so-called two-nucleon plane wave) can be represented as

$$
\begin{gathered}
\left|\mathbf{p} \mu_{1} \mu_{2}, \tau_{1} \tau_{2}\right\rangle=\sum\left(\frac{1}{2} \mu_{1} \frac{1}{2} \mu_{2} \mid S M_{S}\right)\left(\frac{1}{2} \tau_{1} \frac{1}{2} \tau_{2} \mid T M_{T}\right) \\
\left(l m_{l} S M_{S} \mid J M_{J}\right) Y_{l m_{l}}^{*}(\mathbf{p} / p)\left|p J(l S) M_{J}, T M_{T}\right\rangle
\end{gathered}
$$

where $J, S$ and $T$ are, respectively, total angular momentum, spin and isospin of the $N N$ pair, being the eigenvalues of the operators $\mathbf{J}_{\text {ferm }}, \mathbf{S}_{\text {ferm }}$ and $\mathbf{T}_{\text {ferm }}$. Here

$$
\mathbf{J}_{\text {ferm }}=\mathbf{L}_{\text {ferm }}+\mathbf{S}_{\text {ferm }}
$$

where $\mathbf{L}_{\text {ferm }}\left(\mathbf{S}_{\text {ferm }}\right)$ the orbital (spin) momentum of the fermion field,

$$
\begin{aligned}
\mathbf{L}_{\text {ferm }}= & \frac{i}{2} \sum_{\mu} \int d \mathbf{p} \mathbf{p} \times\left[\frac{\partial b_{c}^{\dagger}(\mathbf{p} \mu)}{\partial \mathbf{p}} b_{c}(\mathbf{p} \mu)\right. \\
& -b_{c}^{\dagger}(\mathbf{p} \mu) \frac{\partial b_{c}(\mathbf{p} \mu)}{\partial \mathbf{p}}+\frac{\partial d_{c}^{\dagger}(\mathbf{p} \mu)}{\partial \mathbf{p}} d_{c}(\mathbf{p} \mu) \\
& \left.-d_{c}^{\dagger}(\mathbf{p} \mu) \frac{\partial d_{c}(\mathbf{p} \mu)}{\partial \mathbf{p}}\right]
\end{aligned}
$$

and

$$
\begin{aligned}
\mathbf{S}_{\text {ferm }}=\frac{1}{2} \sum_{\mu \mu^{\prime}} \int d \mathbf{p} \chi_{\mu^{\prime}}^{\dagger} \sigma \chi_{\mu}\left\{b_{c}^{\dagger}\left(\mathbf{p} \mu^{\prime}\right) b_{c}(\mathbf{p} \mu)\right. & \\
& \left.-d_{c}^{\dagger}\left(\mathbf{p} \mu^{\prime}\right) d_{c}(\mathbf{p} \mu)\right\},
\end{aligned}
$$

where $\chi_{\mu^{\prime}}\left(\chi_{\mu}\right)$ are the Pauli spinors. For brevity, we do not show the isospin operator $\mathbf{T}_{\text {ferm }}$.

The corresponding eigenvalue equations look as

$$
\begin{gathered}
\mathbf{J}_{\text {ferm }}^{2}\left|p J(l S) M_{J}\right\rangle=J(J+1)\left|p J(l S) M_{J}\right\rangle \\
J_{\text {ferm }}^{z}\left|p J(l S) M_{J}\right\rangle=M_{J}\left|p J(l S) M_{J}\right\rangle
\end{gathered}
$$

and

$$
\begin{gathered}
\mathbf{S}_{\text {ferm }}^{2}\left|\mathbf{p} S M_{S}\right\rangle=S(S+1)\left|\mathbf{p} S M_{S}\right\rangle \\
S_{\text {ferm }}^{z}\left|\mathbf{p} S M_{S}\right\rangle=M_{S}\left|\mathbf{p} S M_{S}\right\rangle
\end{gathered}
$$

Doing so, we have introduced the vectors ${ }^{2}$

$$
\left|\mathbf{p} S M_{S}\right\rangle=\sum\left(\frac{1}{2} \mu_{1} \frac{1}{2} \mu_{2} \mid S M_{S}\right)\left|\mathbf{p} \mu_{1} \mu_{2}\right\rangle
$$

and

$$
\begin{aligned}
& \left|p J(l S) M_{J}\right\rangle \\
& \quad=\int d \hat{\mathbf{p}} Y_{l m_{l}}(\mathbf{p} / p)\left|\mathbf{p} S M_{S}\right\rangle\left(\operatorname{lm}_{l} S M_{S} \mid J M_{J}\right)
\end{aligned}
$$

A simple way of deriving Eqs.(47)-(48) is to use the transformation

$$
\begin{gathered}
U_{F}^{c}(R)\left|\mathbf{p} S M_{S}\right\rangle=\left|R \mathbf{p} S M_{S}^{\prime}\right\rangle D_{M_{S}^{\prime} M_{S}}^{(S)}(R) \\
\forall R \in \text { the rotation group }
\end{gathered}
$$

One should note that in our case the separable ansatz

$$
\left|\mathbf{p}_{1} \mathbf{p}_{2} \mu_{1} \mu_{2}\right\rangle=\left|\mathbf{p}_{1} \mu_{1}\right\rangle\left|\mathbf{p}_{2} \mu_{2}\right\rangle
$$

often exploited in relativistic quantum mechanics (RQM) (see, e.g., [16] and [17]) does not work. However, one can employ the similarity transformation ${ }^{3}$

$$
\begin{aligned}
U_{F}^{c}(\Lambda, a) b_{c}^{\dagger}(p \mu) & U_{F}^{c} \dagger(\Lambda, a) \\
= & e^{i \Lambda p \cdot a} b_{c}^{\dagger}\left(\Lambda p \mu^{\prime}\right) D_{\mu^{\prime} \mu}^{\left(\frac{1}{2}\right)}(W(\Lambda, p))
\end{aligned}
$$

${ }^{2}$ For a moment, the isospin quantum numbers are suppressed.

${ }^{3}$ Sometimes it is convenient to handle the operators $b_{c}^{\dagger}(p \mu)=$ $\sqrt{p_{0}} b_{c}^{\dagger}(\mathbf{p} \mu)$ and their adjoints $b_{c}(p \mu)$ that meet covariant relations $\left\{b_{c}^{\dagger}\left(p^{\prime} \mu^{\prime}\right), b_{c}(p \mu)\right\}=p_{0} \delta\left(\mathbf{p}^{\prime}-\mathbf{p}\right) \delta_{\mu^{\prime} \mu}$ 
with the Wigner rotation $W(\Lambda, p)$ (e.g., for rotations $W(R, p)=$ $R$ ) and the property of the physical vacuum $\Omega$ to be invariant with respect to unitary transformations $U_{F}^{c}$ in the CPR (some details can be found in a separate paper).

The use of expansion (43) gives rise to the well known JST representation, in which

$$
\begin{array}{r}
\left\langle p^{\prime} J^{\prime}\left(l^{\prime} S^{\prime}\right) M_{J}^{\prime}, T^{\prime} M_{T}^{\prime}|\bar{R}(E)\{\bar{V}\}| p J(l S) M_{J}, T M_{T}\right\rangle \\
=\bar{R}(E)\{\bar{V}\}_{l^{\prime} l}^{J S T}\left(p^{\prime}, p\right) \delta_{J^{\prime} J} \delta_{M_{J}^{\prime} M_{J}} \delta_{S^{\prime} S} \delta_{T^{\prime} T} \delta_{M_{T}^{\prime} M_{T}},
\end{array}
$$

so Eq.(40) reduces to the set of simple integral equations,

$$
\begin{aligned}
\bar{R}_{l^{\prime} l}^{J S T}\left(p^{\prime}, p\right) & =\bar{V}_{l^{\prime} l}^{J S T}\left(p^{\prime}, p\right) \\
+ & \sum_{l^{\prime \prime}} p \cdot v \cdot \int_{0}^{\infty} \frac{q^{2} d q}{2\left(E_{p}-E_{q}\right)} \bar{V}_{l^{\prime} l^{\prime \prime}}^{J S T}\left(p^{\prime}, q\right) \bar{R}_{l^{\prime \prime} l}^{J S T}(q, p)
\end{aligned}
$$

to be solved for each submatrix $\bar{R}^{J S T}$ composed of the elements

$$
\bar{R}_{l^{\prime} l}^{J S T}\left(p^{\prime}, p\right) \equiv \bar{R}_{l^{\prime} l}^{J S T}\left(p^{\prime}, p ; 2 E_{p}\right),
$$

where $E_{p}=\sqrt{\mathbf{p}^{2}+m^{2}}$ the collision energy in the c.m.s.. One should note that in view of the charge independence assumed in this work one has to solve two separate equations for isospin values $T=0$ and $T=1$.

\section{Results of numerical calculations and their discussion}

In the course of our computations we have used the socalled matrix inversion method (MIM) (see [18] and refs. therein). Since we deal with the relativistic dispersion law for the particle energies, the well known substraction procedure within the MIM leads to equations

$$
\begin{aligned}
& R_{l^{\prime} l}^{J S T}\left(p^{\prime}, p\right)=V_{l^{\prime} l}^{J S T}\left(p^{\prime}, p\right) \\
& +\frac{1}{2} \sum_{l^{\prime \prime}} \int_{0}^{\infty} \frac{d q}{p^{2}-q^{2}}\left\{q^{2}\left(E_{p}+E_{q}\right) V_{l^{\prime} l^{\prime \prime}}^{J S T}\left(p^{\prime}, q\right) R_{l^{\prime \prime} l}^{J S T}(q, p)\right. \\
& \left.-2 p^{2} E_{p} V_{l^{\prime} l^{\prime \prime}}^{J S T}\left(p^{\prime}, p\right) R_{l^{\prime \prime} l}^{J S T}(p, p)\right\}
\end{aligned}
$$

To facilitate comparison with some derivations and calculations from Refs. [2], [20], we introduce the notation

$$
\begin{aligned}
& \left\langle\mathbf{p}^{\prime} \mu_{1}^{\prime} \mu_{2}^{\prime}\left|v_{b}^{U C T}\right| \mathbf{p} \mu_{1} \mu_{2}\right\rangle \\
& \quad \equiv-F_{b}^{2}\left(p^{\prime}, p\right)\left[v_{b}\left(1^{\prime}, 2^{\prime} ; 1,2\right)+v_{b}\left(2^{\prime}, 1^{\prime} ; 2,1\right)\right]
\end{aligned}
$$

\begin{tabular}{|c|c|c|c|}
\hline Meson & & Potential B & UCT \\
\hline \multirow[t]{3}{*}{$\pi$} & $g_{\pi}^{2} / 4 \pi$ & 14.4 & 14.5 \\
\hline & $\Lambda_{\pi}$ & 1700 & 2200 \\
\hline & $m_{\pi}$ & 138.03 & 138.03 \\
\hline \multirow[t]{3}{*}{$\eta$} & $g_{\eta}^{2} / 4 \pi$ & 3 & 2.8534 \\
\hline & $\Lambda_{\eta}$ & 1500 & 1200 \\
\hline & $m_{\eta}$ & 548.8 & 548.8 \\
\hline \multirow[t]{4}{*}{$\rho$} & $g_{\rho}^{2} / 4 \pi$ & 0.9 & 1.3 \\
\hline & $\Lambda_{\rho}$ & 1850 & 1450 \\
\hline & $f_{\rho} / g_{\rho}$ & 6.1 & 5.85 \\
\hline & $m_{\rho}$ & 769 & 769 \\
\hline \multirow[t]{3}{*}{$\omega$} & $g_{\omega}^{2} / 4 \pi$ & 24.5 & 27 \\
\hline & $\Lambda_{\omega}$ & 1850 & 2035.59 \\
\hline & $m_{\omega}$ & 782.6 & 782.6 \\
\hline \multirow[t]{3}{*}{$\delta$} & $g_{\delta}^{2} / 4 \pi$ & 2.488 & 1.6947 \\
\hline & $\Lambda_{\delta}$ & 2000 & 2200 \\
\hline & $m_{\delta}$ & 983 & 983 \\
\hline \multirow[t]{3}{*}{$\sigma, T=0$} & $g_{\sigma}^{2} / 4 \pi$ & 18.3773 & 19.4434 \\
\hline & $\Lambda_{\sigma}$ & 2000 & 1538.13 \\
\hline & $m_{\sigma}$ & 720 & 717.7167 \\
\hline \multirow[t]{3}{*}{$\sigma, T=1$} & $g_{\sigma}^{2} / 4 \pi$ & 8.9437 & 10.8292 \\
\hline & $\Lambda_{\sigma}$ & 1900 & 2200 \\
\hline & $m_{\sigma}$ & 550 & 568.8612 \\
\hline
\end{tabular}

for the regularized UCT quasipotentials in the c.m.s. As in Ref.[2], we put that invariants $F_{b}\left(p^{\prime}, p\right)=F_{b}\left(\Lambda p^{\prime}, \Lambda p\right)$ have a phenomenological form,

$$
F_{b}\left(p^{\prime}, p\right)=\left[\frac{\Lambda_{b}^{2}-m_{b}^{2}}{\Lambda_{b}^{2}-\left(p^{\prime}-p\right)^{2}}\right]^{n_{b}} \equiv F_{b}\left[\left(p^{\prime}-p\right)^{2}\right]
$$

Table 1. The best-fit parameters for the two models. The third (fourth) column taken from Table A.1 [20] (obtained by solving Eqs.(56) with a least squares fitting to OBEP values in Table 2). All masses are in $\mathrm{MeV}$, and $n_{b}=1$ except for $n_{\rho}=n_{\omega}=2$.

Doing so, we have

$$
\begin{aligned}
& \left\langle\mathbf{p}^{\prime} \mu_{1}^{\prime} \mu_{2}^{\prime}\left|v_{s}^{U C T}\right| \mathbf{p} \mu_{1} \mu_{2}\right\rangle \\
& \quad=g_{s}^{2} \bar{u}\left(\mathbf{p}^{\prime}\right) u(\mathbf{p}) \frac{F_{s}^{2}\left[\left(p^{\prime}-p\right)^{2}\right]}{\left(p^{\prime}-p\right)^{2}-m_{s}^{2}} \bar{u}\left(-\mathbf{p}^{\prime}\right) u(-\mathbf{p}),
\end{aligned}
$$

$$
\begin{aligned}
& \left\langle\mathbf{p}^{\prime} \mu_{1}^{\prime} \mu_{2}^{\prime}\left|v_{p s}^{U C T}\right| \mathbf{p} \mu_{1} \mu_{2}\right\rangle \\
= & -g_{p s}^{2} \bar{u}\left(\mathbf{p}^{\prime}\right) \gamma_{5} u(\mathbf{p}) \frac{F_{p s}^{2}\left[\left(p^{\prime}-p\right)^{2}\right]}{\left(p^{\prime}-p\right)^{2}-m_{p s}^{2}} \bar{u}\left(-\mathbf{p}^{\prime}\right) \gamma_{5} u(-\mathbf{p})
\end{aligned}
$$

$$
\begin{aligned}
& \left\langle\mathbf{p}^{\prime} \mu_{1}^{\prime} \mu_{2}^{\prime}\left|v_{\mathrm{v}}^{U C T}\right| \mathbf{p} \mu_{1} \mu_{2}\right\rangle=-\frac{F_{\mathrm{v}}^{2}\left[\left(p^{\prime}-p\right)^{2}\right]}{\left(p^{\prime}-p\right)^{2}-m_{\mathrm{v}}^{2}} \\
& \times\left\{\overline { u } ( \mathbf { p } ^ { \prime } ) \left[\left(g_{\mathrm{v}}+f_{\mathrm{v}}\right) \gamma_{v}-\frac{f_{\mathrm{v}}}{2 m}\left(p^{\prime}+p\right)_{v}\right.\right. \\
& \left.-\frac{f_{\mathrm{v}}}{2 m}\left(E_{\mathbf{p}^{\prime}}-E_{\mathbf{p}}\right)\left[\gamma_{0} \gamma_{v}-g_{0 v}\right]\right] u(\mathbf{p}) \\
& \times \bar{u}\left(-\mathbf{p}^{\prime}\right)\left[\left(g_{\mathrm{v}}+f_{\mathrm{v}}\right) \gamma^{v}-\frac{f_{\mathrm{v}}}{2 m} \frac{\left.p^{\prime}+p\right)^{v}}{-\frac{f_{\mathrm{v}}}{2 m}}\left(E_{\mathbf{p}^{\prime}}-E_{\mathbf{p}}\right)\left[\gamma^{0} \gamma^{v}-g^{0 v}\right]\right] u(-\mathbf{p})
\end{aligned}
$$


$19^{\text {th }}$ International IUPAP Conference on Few-Body Problems in Physics

Table 2. Neutron-proton phase shifts (in degrees) for various laboratory energies (in MeV). The OBEP(OBEP*)-rows taken from Table 5.2 [20] (calculated by solving Eqs.(61) with the model parameters from the third column in Table 1). The UCT ${ }^{*}(\mathrm{UCT})-$ rows calculated by solving Eqs.(56) with the parameters from the third (fourth) column in Table 1. As in [2], we have used the bar convention [19] for the phase parameters.

\begin{tabular}{|c|c|c|c|c|c|c|c|}
\hline State & Potential & 25 & 50 & 100 & 150 & 200 & 300 \\
\hline \multirow{4}{*}{${ }^{1} S_{0}$} & OBEP & 50.72 & 39.98 & 25.19 & 14.38 & 5.66 & -8.18 \\
\hline & OBEP* & 50.71 & 39.98 & 25.19 & 14.37 & 5.66 & -8.18 \\
\hline & $\mathrm{UCT}^{*}$ & 66.79 & 53.01 & 36.50 & 25.27 & 16.54 & 3.12 \\
\hline & UCT & 50.03 & 39.77 & 25.55 & 15.20 & 6.92 & -6.07 \\
\hline \multirow{4}{*}{${ }^{1} P_{1}$} & OBEP & -7.21 & -11.15 & -16.31 & -20.21 & -23.47 & -28.70 \\
\hline & OBEP* & -7.17 & -11.15 & -16.32 & -20.21 & -23.48 & -28.71 \\
\hline & $\mathrm{UCT}^{*}$ & -7.40 & -11.70 & -17.73 & -22.63 & -26.98 & -34.54 \\
\hline & UCT & -7.15 & -10.95 & -15.62 & -18.90 & -21.49 & -25.41 \\
\hline \multirow{4}{*}{${ }^{1} D_{2}$} & OBEP & 0.68 & 1.58 & 3.34 & 4.94 & 6.21 & 7.49 \\
\hline & OBEP* & 0.68 & 1.58 & 3.34 & 4.94 & 6.21 & 7.49 \\
\hline & $\mathrm{UCT}^{*}$ & 0.68 & 1.59 & 3.40 & 5.10 & 6.52 & 8.20 \\
\hline & UCT & 0.68 & 1.56 & 3.22 & 4.68 & 5.77 & 6.68 \\
\hline \multirow{4}{*}{${ }^{3} P_{0}$} & OBEP & 9.34 & 12.24 & 9.80 & 4.57 & -1.02 & -11.48 \\
\hline & OBEP* & 9.34 & 12.24 & 9.80 & 4.57 & -1.02 & -11.48 \\
\hline & $\mathrm{UCT}^{*}$ & 9.48 & 12.53 & 10.32 & 5.27 & -0.15 & -10.27 \\
\hline & UCT & 9.30 & 12.16 & 9.81 & 4.73 & -0.68 & -10.76 \\
\hline \multirow{4}{*}{${ }^{3} P_{1}$} & OBEP & -5.33 & -8.77 & -13.47 & -17.18 & -20.49 & -26.38 \\
\hline & OBEP* & -5.33 & -8.77 & -13.47 & -17.18 & -20.48 & -26.38 \\
\hline & $\mathrm{UCT}^{*}$ & -5.27 & -8.62 & -13.09 & -16.56 & -19.63 & -25.06 \\
\hline & UCT & -5.28 & -8.58 & -12.85 & -16.06 & -18.86 & -23.79 \\
\hline \multirow{4}{*}{${ }^{3} D_{2}$} & OBEP & 3.88 & 9.29 & 17.67 & 22.57 & 24.94 & 25.36 \\
\hline & OBEP* & 3.89 & 9.29 & 17.67 & 22.57 & 24.94 & 25.36 \\
\hline & $\mathrm{UCT}^{*}$ & 3.86 & 9.15 & 17.12 & 21.51 & 23.47 & 23.48 \\
\hline & UCT & 3.89 & 9.25 & 17.31 & 21.77 & 23.75 & 23.61 \\
\hline \multirow{4}{*}{${ }^{3} S_{1}$} & OBEP & 80.32 & 62.16 & 41.99 & 28.94 & 19.04 & 4.07 \\
\hline & OBEP* & 80.31 & 62.15 & 41.98 & 28.93 & 19.03 & 4.06 \\
\hline & UCT $^{*}$ & 92.30 & 72.71 & 51.44 & 38.10 & 28.20 & 13.70 \\
\hline & UCT & 79.60 & 61.53 & 41.57 & 28.75 & 19.08 & 4.60 \\
\hline \multirow{4}{*}{${ }^{3} D_{1}$} & OBEP & -2.99 & -6.86 & -12.98 & -17.28 & -20.28 & -23.72 \\
\hline & OBEP ${ }^{*}$ & -2.99 & -6.87 & -12.99 & -17.28 & -20.29 & -23.72 \\
\hline & UCT $^{*}$ & -2.74 & -6.43 & -12.36 & -16.54 & -19.47 & -22.78 \\
\hline & UCT & -3.00 & -6.90 & -13.12 & -17.66 & -21.11 & -26.03 \\
\hline \multirow{4}{*}{$\varepsilon_{1}$} & OBEP & 1.76 & 2.00 & 2.24 & 2.58 & 3.03 & 4.03 \\
\hline & OBEP* & 1.76 & 2.00 & 2.24 & 2.58 & 3.03 & 4.03 \\
\hline & UCT $^{*}$ & 0.02 & -0.12 & -0.17 & 0.04 & 0.41 & 1.40 \\
\hline & UCT & 1.80 & 2.01 & 2.19 & 2.50 & 2.90 & 3.83 \\
\hline \multirow{4}{*}{${ }^{3} P_{2}$} & OBEP & 2.62 & 6.14 & 11.73 & 14.99 & 16.65 & 17.40 \\
\hline & OBEP* & 2.62 & 6.14 & 11.73 & 14.99 & 16.65 & 17.39 \\
\hline & $\mathrm{UCT}^{*}$ & 2.80 & 6.61 & 12.71 & 16.28 & 18.10 & 18.91 \\
\hline & UCT & 2.57 & 6.00 & 11.32 & 14.18 & 15.37 & 15.07 \\
\hline \multirow{4}{*}{${ }^{3} F_{2}$} & OBEP & 0.11 & 0.34 & 0.77 & 1.04 & 1.10 & 0.52 \\
\hline & OBEP* & 0.11 & 0.34 & 0.77 & 1.04 & 1.10 & 0.52 \\
\hline & UCT $^{*}$ & 0.11 & 0.34 & 0.77 & 1.05 & 1.13 & 0.64 \\
\hline & UCT & 0.11 & 0.34 & 0.75 & 1.00 & 1.03 & 0.41 \\
\hline \multirow{4}{*}{$\varepsilon_{2}$} & OBEP & -0.86 & -1.82 & -2.84 & -3.05 & -2.85 & -2.02 \\
\hline & OBEP* & -0.86 & -1.82 & -2.84 & -3.05 & -2.85 & -2.02 \\
\hline & UCT* $^{*}$ & -0.87 & -1.83 & -2.82 & -2.99 & -2.75 & -1.88 \\
\hline & UCT & -0.86 & -1.83 & -2.84 & -3.05 & -2.89 & -2.18 \\
\hline
\end{tabular}



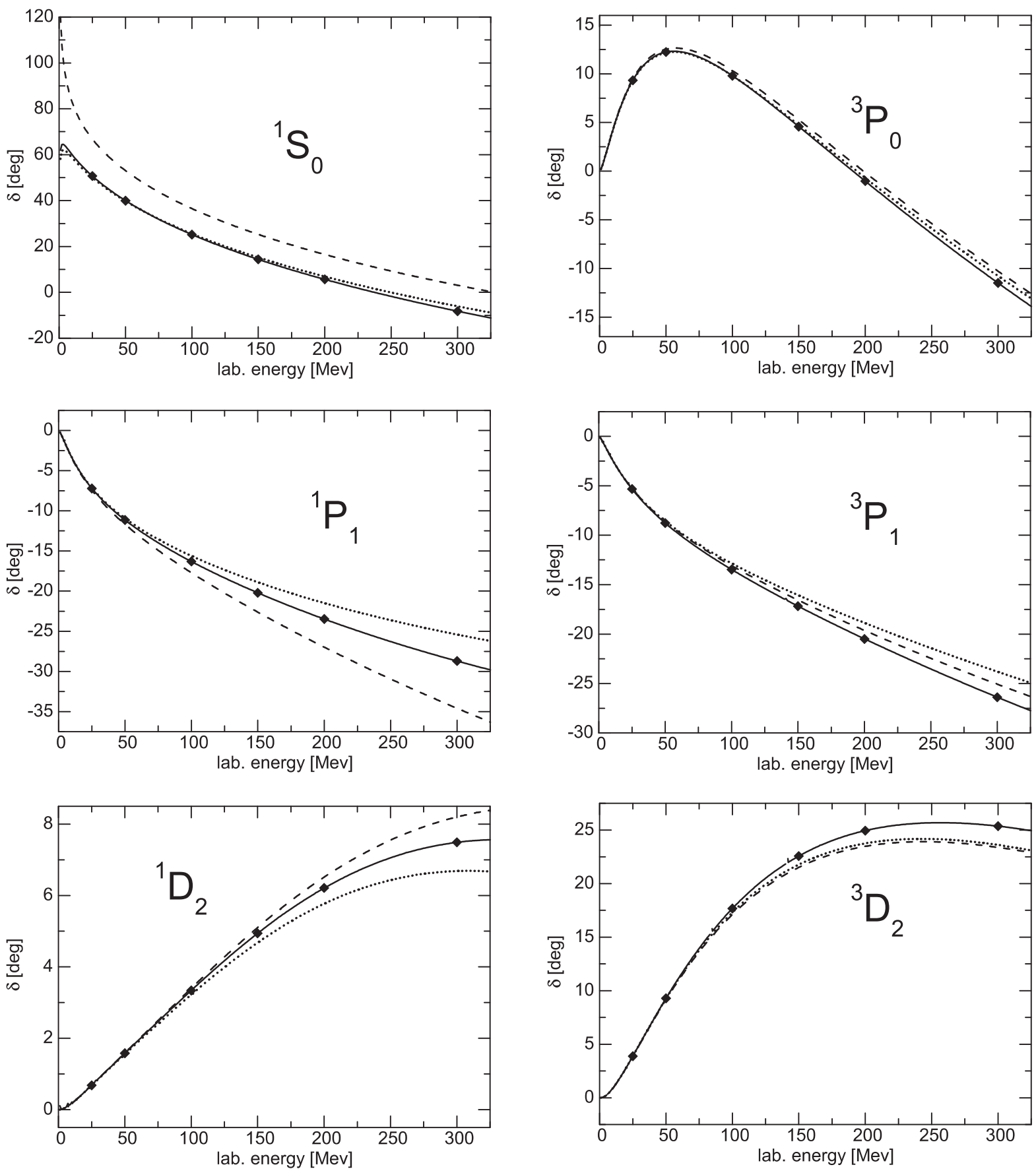

Fig. 2. Neutron-proton phase parameters for the uncoupled partial waves, plotted versus the nucleon kinetic energy in the lab. system. Dashed[solid] curves calculated with Potential B parameters (Table 1) by solving Eqs. (56)[(61)]. Dotted represent the solutions of Eqs. (56) with UCT parameters (Table 1). The rhombs show original OBEP results (see Table 2).

$$
\begin{aligned}
-\frac{f_{\mathrm{v}}^{2}}{4 m^{2}}\left(E_{p^{\prime}}-E_{p}\right)^{2} & \bar{u}\left(\mathbf{p}^{\prime}\right)\left[\gamma_{0} \gamma_{v}-g_{0 v}\right] u(\mathbf{p}) \\
& \left.\times \bar{u}\left(-\mathbf{p}^{\prime}\right)\left[\gamma^{0} \gamma^{v}-g^{0 v}\right] u(-\mathbf{p})\right\},
\end{aligned}
$$

where $\left.\overline{\left(p^{\prime}+p\right)}\right)^{v}=\left(E_{\mathbf{p}^{\prime}}+E_{\mathbf{p}},-\left(\mathbf{p}^{\prime}+\mathbf{p}\right)\right)$.

At first sight, such a regularization can be achieved via a simple substitution $g_{b} \rightarrow g_{b} F_{b}\left(p^{\prime}, p\right)$ with some cutoff functions $F_{b}\left(p^{\prime}, p\right)$ depending on the 4-momenta $p^{\prime}$ and $p$.
However, the principal moment is to satisfy the requirement (22) for the Hamiltonian invariant under space inversion, time reversal and charge conjugation. In this context, let us remind that the baryon-nucleon-nucleon form factors are expressed through the matrix elements $\left\langle p^{\prime}\left|j_{b}(0)\right| p\right\rangle$ of the corresponding baryon current density $j_{b}(x)$ at $x=0$ between physical(clothed) one-nucleon states [21]. Such matrix elements might be evaluated in terms of the cutoffs 
$19^{\text {th }}$ International IUPAP Conference on Few-Body Problems in Physics
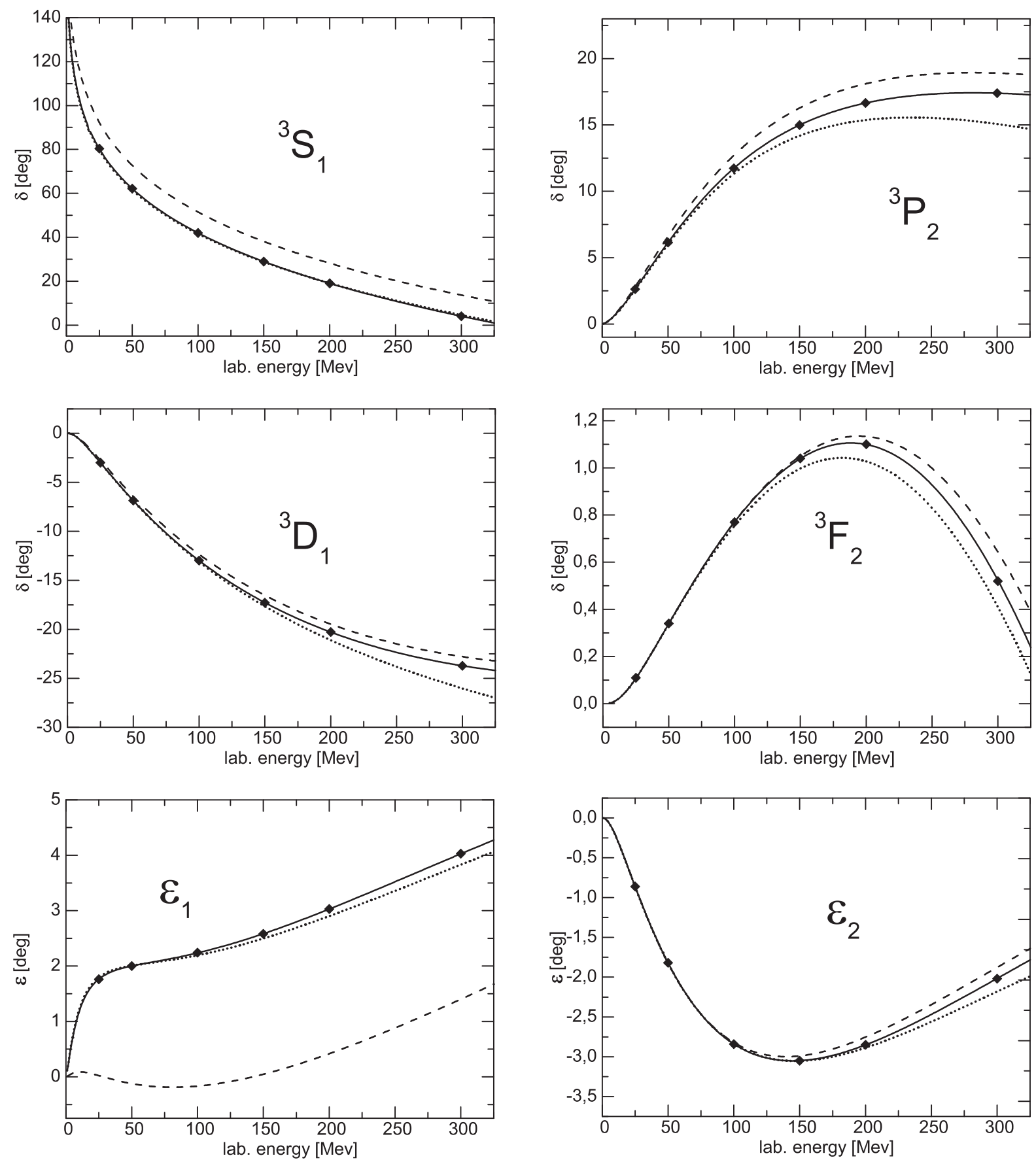

Fig. 3. The same in Fig. 2 but for the coupled waves.

$F_{b}\left(p^{\prime}, p\right)$ using some idea from [22] (cf. the clothed particle representation of a current therein).

Replacing in equations (57)-(59)

$$
\frac{1}{\left(p^{\prime}-p\right)^{2}-m_{b}^{2}} F_{b}^{2}\left[\left(p^{\prime}-p\right)^{2}\right]
$$

by

$$
\frac{-1}{\left(\mathbf{p}^{\prime}-\mathbf{p}\right)^{2}+m_{b}^{2}} F_{b}^{2}\left[-\left(\mathbf{p}^{\prime}-\mathbf{p}\right)^{2}\right]
$$

and neglecting the tensor-tensor term

$$
\begin{aligned}
& \frac{f_{\mathrm{v}}^{2}}{4 m^{2}}\left(E_{p^{\prime}}-E_{p}\right)^{2} \\
& \times \bar{u}\left(\mathbf{p}^{\prime}\right)\left[\gamma_{0} \gamma_{v}-g_{0 v}\right] u(\mathbf{p}) \bar{u}\left(-\mathbf{p}^{\prime}\right)\left[\gamma^{0} \gamma^{v}-g^{0 v}\right] u(-\mathbf{p})
\end{aligned}
$$

in (59), we obtain approximate expressions that with the common factor

$$
(2 \pi)^{-3} m^{2} / E_{p^{\prime}} E_{p}
$$

instead of

$$
(2 \pi)^{-3} m / \sqrt{E_{p^{\prime}} E_{p}}
$$


are equivalent to Eqs. (E.21)-(E.23) from [2]. Such an equivalence becomes coincidence if in our formulae instead of the canonical two-nucleon basis $\left|\mathbf{p} \mu_{1} \mu_{2}\right\rangle$ one uses the helicity basis as in [2].

In the context, we have considered the set of equations

$$
\begin{aligned}
& { }^{B} R_{l^{\prime} l}^{J S T}\left(p^{\prime}, p\right)={ }^{B} V_{l^{\prime} l}^{J S T}\left(p^{\prime}, p\right) \\
& +m \sum_{l^{\prime \prime}} \int_{0}^{\infty} \frac{d q}{p^{2}-q^{2}}\left\{q^{2 B} V_{l^{\prime} l^{\prime \prime}}^{J S T}\left(p^{\prime}, q\right)^{B} R_{l^{\prime \prime} l}^{J S T}(q, p)\right. \\
& \left.\quad-p^{2}{ }^{B} V_{l^{\prime} l^{\prime \prime}}^{J S T}\left(p^{\prime}, p\right)^{B} R_{l^{\prime \prime} l}^{J S T}(p, p)\right\},
\end{aligned}
$$

where the superscript $B$ refers to the partial matrix elements of the potential $B$ defined in [20] with the just mentioned interchange of the bases.

Our calculations of the $R$ matrices that meet the equations (56) and (61) are twofold. On the one hand, we will check reliability of our numerical procedure (in particular, its code). On the other hand, we would like to show similarities and discrepancies between our results and those by the Bonn group both on the energy shell and beyond it. These results are depicted in Figs. $2-3$ and collected in Table 2.

As seen in Figs. 2-3, the most appreciable distinctions between the UCT and OBEP curves take place for the phase shifts with the lowest $l$-values. As the orbital angular momentum increases the difference between the solid and dashed curves decreases. Such features may be explained if one takes into account that the approximations under consideration affect mainly high-momentum components of the UCT quasipotentials (their behavior at "small" distances). With the $l$-increase the influence of small distances is suppressed by the centrifugal barrier repulsion.

Of course, it would be more instructive to compare the corresponding half-off-energy-shell $R$-matrices (see definition (55)). Their $p^{\prime}$-dependencies not shown here have been prepared for a separate publication. They are necessary to know when calculating the $\psi^{( \pm)}$scattering states for a two-nucleon system. In the context, one should emphasize that hitherto we have explored the OBEP and UCT $R$-matrices in the c.m.s., where the both approaches yield most close results. It is not the case in those situations when the c.m.s. cannot be referred to everywhere (e.g., in the reactions $N N \rightarrow \gamma N N$ and $\gamma d \rightarrow p n)$. In this respect our studies of the differences between UCT and OBE approaches are under way.

\section{Summary}

The present work has been made to develop a consistent field-theoretical approach in the theory of nucleon-nucleon scattering. It has been shown that the method of UCT's, based upon the notion of clothed particles, is proved to be appropriate in achieving this purpose.

Using the unitary equivalence of the CPR to the BPR, we have seen how in the approximation $K_{I}=K_{I}^{(2)}$ the extremely complicated scattering problem in QFT can be reduced to the three-dimensional LS-type equation for the
$T$-matrix in momentum space.The equation kernel is given by the clothed two-nucleon interaction of the class [2.2]. Such a conversation becomes possible owing to the property of $K_{I}^{(2)}$ to leave the two-nucleon sector and its separate subsectors to be invariant.

Special attention has been paid to the elimination of auxiliary field components. We encounter such a necessity for interacting vector and fermion fields when in accordance with the canonical formalism the interaction Hamiltonian density embodies not only a scalar contribution but nonscalar terms too. It has proved (at least, for the primary $\rho N$ and $\omega N$ couplings) that the UCT method allows us to remove such noncovariant terms directly in the Hamiltonian. To what extent this result will take place in higher orders in coupling constants it will be a subject of further explorations.

\section{References}

1. M. Lacombe et al., Phys. Rev. C21, (1980) 861

2. R. Machleidt, K. Holinde and C. Elster, Phys. Rep. 149, (1987) 1

3. V.G.J. Stocks et al., Phys. Rev. C49, (1994) 2950

4. R.B. Wiringa, V.G.J. Stocks and R. Schiavilla, Phys. Rev. C51, (1995) 38

5. R. Machleidt, Phys. Rev. C63, (2001) 024001

6. F. Gross and A. Stadler, Few Body Syst. 44, (2008) 295

7. C. Ordonez, L. Ray and U. van Kolck, Phys. Rev. Lett. 72, (1994) 1982

8. E. Epelbaum, W. Glöckle and U.-G. Meissner, Nucl. Phys. A671, (2000) 295

9. E. Epelbaum, Prog. Part. Nucl. Phys. 57, (2006) 654

10. A.V. Shebeko and M.I. Shirokov, Phys. Part. Nucl. 32, (2001) 31

11. V. Korda, L. Canton and A. Shebeko, Ann. Phys. 322, (2007) 736

12. S. Weinberg, The Quantum Theory of Fields, (University Press, Cambridge, 1995) Vol. 1

13. D.A. Schütte, Nucl. Phys. A221, (1974) 450

14. K. Tamura, T. Niva, T. Sato and H. Ohtsubo, Prog. Theor. Phys. 80, (1988) 138.

15. A.V. Shebeko, Nucl. Phys. A737, (2004) 252

16. J.Werle, Relativistic Theory of Reactions (PWN - Polish Scientific Publishers, Warszawa, 1966)

17. B.D.Keister, W.N.Polyzou, Adv. Nucl. Phys. 20, (1991) 266

18. G.E. Brown and A.D Jackson, Nucleon-Nucleon Interaction (Amsterdam: North-Holland Publ. Co. 1976)

19. H.Stapp et al., Phys. Rev. 105, (1957) 302

20. R. Machleidt, Adv. Nucl. Phys. 19, (1989) 189

21. S. Gasiorowicz, Elementary Particle Physics, (John Wiley \& Sons, New York, 1966)

22. A.V. Shebeko and M.I. Shirokov, Prog. Part. Nucl. Phys. 44, (2000) 75. 